\title{
Working out working memory
}

How information is retained in working memory (WM) - particularly when it is not being directly attended but may still be potentially relevant - is not clear. WM has long been proposed to depend on persistent increases in neural activity; however, some recent studies instead suggest that non-attended WM information can be held by synaptic mechanisms independent of sustained activity, until it is needed. Postle and colleagues now report findings to support this latter model of WM: they find that unattended information can lie latent in WM and then be reactivated by transcranial magnetic stimulation (TMS).

In this study, participants performed a WM task. In each trial, two sample stimuli (for example, a face and a word) were presented together. Next, a first cue (C1) indicated which of the two samples (for example, the face) would need to be attended for a mental comparison with a subsequently presented test (T1) stimulus; for example, participants had to judge whether the $\mathrm{T} 1$ and sample faces were the same or different. After the response to $\mathrm{T} 1$, a subsequent cue, $\mathrm{C} 2$, indicated whether the same stimulus (the original face) or the other sample stimulus (the word) would need to be compared with a second test stimulus, T2. Different stimulus categories produced different patterns of brain activity detected by functional MRI (fMRI) and electroencephalography (EEG), and so these patterns could be used to interpret the brain's representation of the sample stimuli in WM and to guide TMS stimulation.

Multivariate pattern analysis of participants' fMRI responses revealed that, immediately after $\mathrm{C} 1$, the amount of coded information relating to the unattended sample stimulus (called the 'unattended memory item' (UMI)) dropped to baseline. However, if $\mathrm{C} 2$ indicated that the UMI should be reattended, the amount of coded information relating to this stimulus increased again. Thus, UMIs can be held in a latent state and subsequently reinstated.

Next, the authors recorded participants' EEG responses during the task and tested the effects of a single TMS pulse to category-preferring regions of the brain, 2-3 s after C1. Strikingly, TMS led to a transient increase in the EEG representation of the unattended stimulus - a 'reactivation' of the UMI. Moreover, TMS had a similar effect even if it was applied to a brain region preferring a category different to that of the unattended stimulus' category. This suggests that TMS may reactivate UMIs by stimulating UMI- and trial-relevant cortical networks.

In the WM task, if a stimulus is not cued by $\mathrm{C} 2$, it is no longer relevant to the trial. Whereas TMS to a category-general area after $\mathrm{C} 1$ reactivated UMIs, TMS after C2 had no such effect on the newly irrelevant memory items. This observation indicates that UMIs can be cached in a distinct state to irrelevant memories.

Last, the authors tested whether the TMS reactivation of a UMI would induce an artificial increase in attention towards the unattended stimulus. In a slightly different version of the WM task, $20 \%$ of the test stimuli were changed to stimuli from the same test category as the UMI, as 'lures'. TMS after C1 increased the likelihood that participants incorrectly responded to lure $\mathrm{T} 1$ stimuli, but TMS after C2 did not increase incorrect responses to lure T2 stimuli. Thus, UMIs, but not irrelevant memory items, can be brought back into the focus of attention.

These findings suggest that working memories for unattended items can be maintained without an increase in activity, and can be reactivated again at a later point.

Natasha Bray

ORIGINAL ARTICLE Rose, N. S. et al. Reactivation of latent working memories with transcranial magnetic stimulation. Science 354, 1136-1139 (2016)
TMS led to

a transient

increase

in the EEG representation of the unattended stimulus

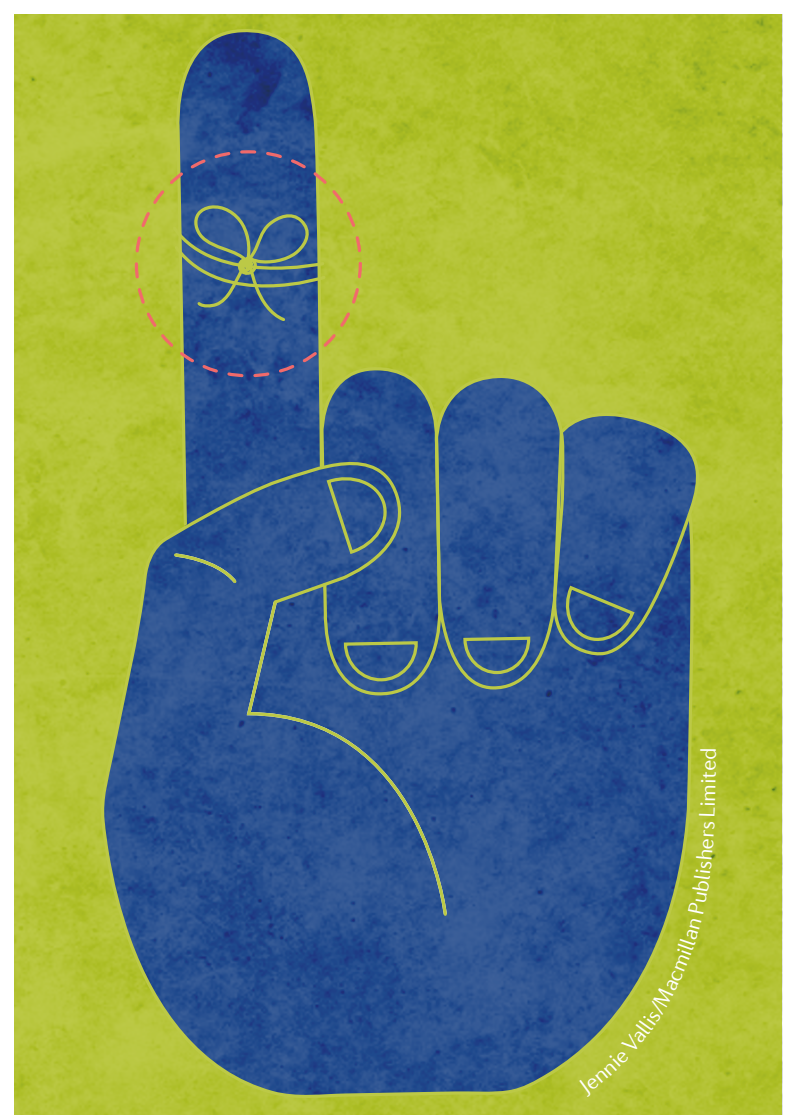

